\title{
A stop codon-dependent internal secondary translation initiation region in Escherichia coli rpoS
}

\author{
POCHI RAMALINGAM SUBBARAYAN and MALANCHA SARKAR \\ Department of Medicine, University of Miami School of Medicine, Miami, Florida 33136, USA
}

\begin{abstract}
Sigma $S\left(\sigma^{s}\right)$ encoded by rpoS is a stationary phase-specific $\sigma$ subunit of the Escherichia coli RNA polymerase holoenzyme. In many $E$. coli strains, rpoS has an amber stop as codon 33 (rpoSAm), resulting in a 32-amino-acid-long peptide. Nevertheless, suppressor-free rpoSAm strains have functional $\sigma^{\mathrm{s}}$. This led us to hypothesize the presence of an intracistronic secondary translational initiation region (STIR) in the E. coli rpoS gene. Here, we demonstrate that the STIR is functional and is controlled by the upstream amber stop codon 33. Removal of the primary translational initiation region did not abolish translation from STIR, ruling out translational coupling. Importantly, the functional STIR conferred survival advantage. Taken together, our results reveal a hitherto unknown physiologically significant post-transcriptional process in E. coli rpoSAm strains.
\end{abstract}

Keywords: STIR; $\sigma^{\text {s }}$; GASP; rpoS; intracistronic translation initiation; ribosome binding site

\section{INTRODUCTION}

In Escherichia coli, translation is initiated from a well-defined translation initiation region (TIR). Typically it consists of a ribosome binding site (RB), initiator codon, and a spacer region (Shine and Dalgarno 1975). E. coli mRNAs exist either in mono- or polycistronic forms. Each cistron of a polycistronic message has its own TIR. Alternately, cistrons may also be translationally coupled wherein a single TIR controls multiple cistrons (Inokuchi et. al. 2000). On the other hand, monocistronic messages typically have a single TIR. However, they can also harbor more than one functional TIR (Laursen et. al. 2002). Recently, we showed that monocistronic rpoS mRNA has two translation initiation regions (Subbarayan and Sarkar 2004b).

In E. coli, DNA-dependent RNA polymerase constitutes transcription machinery. It exists as a core enzyme containing two $\alpha$ and two $\beta$ subunits $\left(\alpha_{2} \beta \beta^{\prime}\right)$. The core enzyme is the catalytic machinery that binds DNA nonspecifically. In association with a $\sigma$ subunit, it forms the holoenzyme. There are seven $\sigma$ subunits in E. coli, and they provide the core form specific promoter recognition activity to initiate transcription (Ishihama 2000). Thus, $\sigma$ subunits are essen-

Reprint requests to: Pochi Ramalingam Subbarayan, Department of Medicine (D8-4), University of Miami School of Medicine, 1550 NW 10th Avenue, Fox 431A, Miami, FL 33136, USA; e-mail: spochi@med.miami. edu; fax: (305) 243-6650.

Article and publication are at http://www.rnajournal.org/cgi/doi/ 10.1261/rna.7500604. tial for transcription initiation in E. coli. One of the $\sigma$ subunits, for example, $\sigma^{\mathrm{S}}$, encoded by rpos (Mulvey and Loewen 1989), governs the expression of many stationary phase-induced and osmotically regulated genes. Hitherto, what is known as wild-type rpoS codes a protein of 330 amino acids. Null mutants of $\sigma^{\mathrm{s}}$ exhibit pleiotropic phenotype under certain growth and stress conditions (Lange and Hengge-Aronis 1991). E. coli rpoS is highly polymorphic. One of the common mutations observed in the rpoS gene is a C-T transition at position 97 (Atlung et. al. 2002; Subbarayan and Sarkar 2004a). This results in an amber codon (CAG-TAG; rpoSAm). Consequently, $\sigma^{\mathrm{S}}$ is prematurely terminated, and suppressor-free rpoSAm mutants would have no $\sigma^{\mathrm{S}}$ activity. However, several rpoSAm strains exhibit reduced $\sigma^{S}$ activity (Subbarayan and Sarkar 2004a). Hydrogen peroxidase II (Catalase II encoded by katE) is regulated by $\sigma^{\mathrm{S}}$, and traditionally, catalase assays have been used as a measure of $\sigma^{\mathrm{S}}$ activity. In fact, the defects in the $\operatorname{rpoS}$ genes of many strains were detected by catalase assay. For instance, rpoSAm strain W3350, has 50\% less catalase II activity than ZK126, a strain expressing full-length $\sigma^{\mathrm{S}}$. Reduction, instead of abrogation of $\sigma^{\mathrm{S}}$-mediated catalase II activity in suppressor-free rpoSAm strains led us to propose translation reinitiation from a strategically placed secondary translation initiation region (STIR) within the rpoS gene (Subbarayan and Sarkar 2004b).

In this report, by site-directed mutagenesis, immunodetection, and catalase assay, we confirm the presence of STIR in the rpoS gene. Our results also demonstrate the depen- 


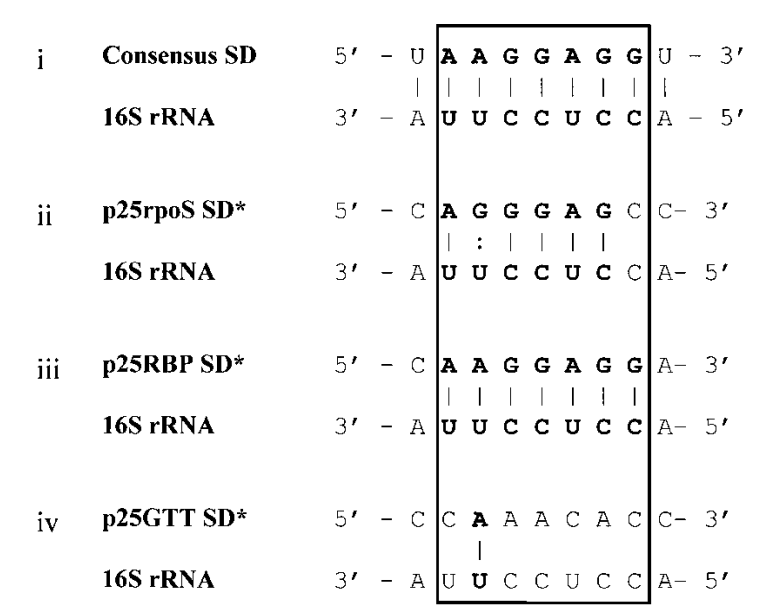

FIGURE 1. Alignment of SD sequence with $3^{\prime}$ end of $16 \mathrm{~S}$ rRNA. (i) Consensus SD sequence with the $3^{\prime}$ end of $16 \mathrm{~S}$ rRNA. (ii) The SD* sequence of $r p o S A m$ base pairs with five of seven bases at the $3^{\prime}$ end of the $16 \mathrm{~S}$ rRNA. (iii) In $p 25 R B P$, the $\mathrm{SD}^{*}$ sequence was mutated to the consensus SD sequence. (iv) In p25GTT, the $\mathrm{SD}^{*}$ sequence was abolished by mutation. $\left(^{*}\right)$ Second or alternate SD. The SD and its complementary sequence at the $3^{\prime}$ end of $16 \mathrm{~S}$ rRNA are boxed.

dence of STIR on an upstream translation stop codon. Removal of the primary TIR did not abolish translation from the STIR, thus ruling out possible translation coupling. Coculture experiments with E. coli strain ZK126 expressing full-length $\sigma^{\mathrm{S}}$ highlight the GASP phenotype of the rpoSAm strain W3350. Collectively, our results provide important insights into a hitherto unknown physiologically relevant post-transcriptional process in the E. coli rpoSAm gene.

\section{RESULTS AND DISCUSSION}

Our earlier studies on the E. coli rpoS gene amply demonstrated the presence of a functional internal STIR (Subbarayan and Sarkar 2004b). In the E. coli rpoS, the ShineDalgarno (SD) sequence AGGGAG and the alternate start codon GUG, with a spacing of 15 nucleotides, constitute the STIR (Subbarayan and Sarkar 2004b). The sequence AGG GAG, found in rpoS could base pair with five of seven bases at the $3^{\prime}$ end of the $16 \mathrm{~S}$ rRNA (Fig. 1ii). About $1.5 \%$ of the 4122 genes have a spacing of 15 in their TIR (Shultzaberger et. al. 2001). To analyze functionality of the STIR, we cloned the mutant rpoSAm gene (NCBI Acc. No. AY142205) in a pBR322-based vector p25rpoSAm. The rpoSAm gene was placed under the direct control of lac $\mathrm{P} / \mathrm{O}$, so that its expression could be regulated (Subbarayan and Sarkar 2004b)

\section{Mutations in STIR affect translation}

SD sequence (Fig. 1i) and the initiator codons have strong effects on translation (Barrick et. al. 1994). Therefore, the STIR region was sequentially mutated, and the mutant plasmids were transformed into the rpoS null mutant ZK1000. Immunoblot and catalase assays were performed with the cell extracts of the transformants.

In the plasmid p25rpoSAm, the STIR was abolished to obtain p25RBA.GTT (Table 1; Fig. 1iv). We could detect neither the truncated $\sigma^{\mathrm{S}}$ (Fig. 2, lane 8) nor the catalase II activity in the transformant of this mutant plasmid (Table 2). The residual $2 \%$ catalase II activity observed might be due to incomplete heat inactivation of catalase I or to some read through of the amber stop. UAG stop codons are known to be particularly leaky, in some cases, read through

TABLE 1. List of mutagenic primers used and properties of the resultant mutant constructs

\begin{tabular}{|c|c|c|c|}
\hline Plasmid template & Sequence $\left(5^{\prime}-3^{\prime}\right)$ & $\begin{array}{l}\text { Primer } \\
\text { length }\end{array}$ & $\begin{array}{l}\text { Properties } \\
\text { conferred }\end{array}$ \\
\hline \multicolumn{4}{|c|}{ Modifications in STIR } \\
\hline p25rpoSAm & 129-GGAACTGTTATCGCAGGGAGCCACACAGCGTGTGTTGGACGCGAC-173 & - & \\
\hline P25ATG & 129-GGAACTGTTATCGCAGGGAGCCACACAGCGTaTGTTGGACGCGAC-173 & 29 mer & GAG-ATG \\
\hline$p 25 R B P$ & 129-GGAACTGTTATCGaaaggaggaACACAGCGTGTGTTGGACGCGAC-173 & 30 mer & $\begin{array}{l}\text { Strengthened } \\
\text { SD sequence }\end{array}$ \\
\hline p25ATG.RBP & 129-GGAACTGTTATCGaaaggaggaACACAGCGTaTGTTGGACGCGAC-173 & - & Strengthened STIR \\
\hline p25GTT & 145-GGAGCCACACAGCGTGT $\boldsymbol{t T T G G A C G C G A C - 1 7 3 ~}$ & 29 mer & GTG-GTT \\
\hline$p 25 R B A$ & 129-GGAACTGTTATCGC $\overline{\text { caaacaCCACACAGCG-158 }}$ & 30 mer & RB Abolished \\
\hline p25RBA.GTT & $\begin{array}{c}\text { 129-GGAACTGTTATCGCAGGGAGCCACACAGCGTGT } \boldsymbol{f T T G G A C G C G A C - 1 7 3} \\
\text { Codon } 33\end{array}$ & - & Abrogated STIR \\
\hline \multicolumn{4}{|c|}{ 84-GGCCTTAGTAGAATAGGAACCCAGTGATAACG-115 } \\
\hline p25CAG & 84-GGCCTTAGTAGAAcAGGAACCCAGTGATAACG-115 & 32 mer & TAG-CAG \\
\hline p25 CAG.RBP.ATG & 21-ATCACGGGTAGGAGCCACCGTATGAGTCAGAATACG-15 & - & \\
\hline p25 5 TIR.CAG.RBP.ATG & 21-ATCACGGGTcaacaCCACCGTetGAGTCAGAATACG-15 & 36 mer & TIR deleted \\
\hline
\end{tabular}

p25rpoSAm is the primary template used to obtain single STIR variants. Multiple mutants were obtained using the immediate respective plasmids as templates. The underlined sequences are the primers. Small bold italics indicate the base changes introduced through mutagenic primers. Bold capital sequences are the internal STIR, codon 33 or the primary TIR of the E. coli rpoS. 
occurs at a frequency as high as $10^{-2}$, probably by tRNA ${ }^{\text {Glu }}$ (Parker 1989; Subbarayan and Deutscher 2001). This confirms STIR as the region from where translation of $\Delta 1-53 \sigma^{\mathrm{S}}$ is initiated in rpoSAm strains.

To evaluate the role of the individual regions/nucleotides within the STIR, we prepared several mutants that carry single or multiple changes in the STIR. Plasmid p25ATG (Table 1) was derived by substitution GTG initiation codon in the STIR to ATG, the more common initiator codon. In the transformants of this mutant, catalase II activity increased by 1.6 -fold, and the $\Delta 1-53 \sigma^{\mathrm{S}}$ quantity by 2.5 -fold (Fig. 2; Table 2).

SD sequence of the STIR was mutated to AAGGAGG to more closely reflect the SD consensus in p25RBP (Fig. 1iii). The catalase II activity increased by about 4.7 -fold (Table 2). About 11-fold more protein was obtained (Fig. 2, lane 4; Table 2). Translation of the truncated $\sigma^{\mathrm{S}}$ increased by 20 fold in plasmid p25RBP.ATG (Fig. 2, lane 5; Table 2), which combined the features of the above two plasmids. Catalase II activity also increased by about 10-fold of p25rpoSAm (Table 2).

\section{Activation of STIR depends on premature (primary) translation termination}

In the derivative plasmid $p 25 C A G$, the TAG amber codon of p25rpoSAm was changed to CAG. Elimination of the stop codon restored the full-length protein (Fig. 2, lane 7). It also increased catalase II activity by about ninefold and quantity by 5.4 -fold (Table 2 ). We could not detect the N-terminaltruncated $\sigma^{\mathrm{S}}$, namely, $\Delta 1-53 \sigma^{\mathrm{S}}$, from the transformants of p25CAG. Therefore, in the absence of premature translation termination at the codon 33 as in rpoSAm, the ribosomes rapidly move forward and cover the downstream Secondary

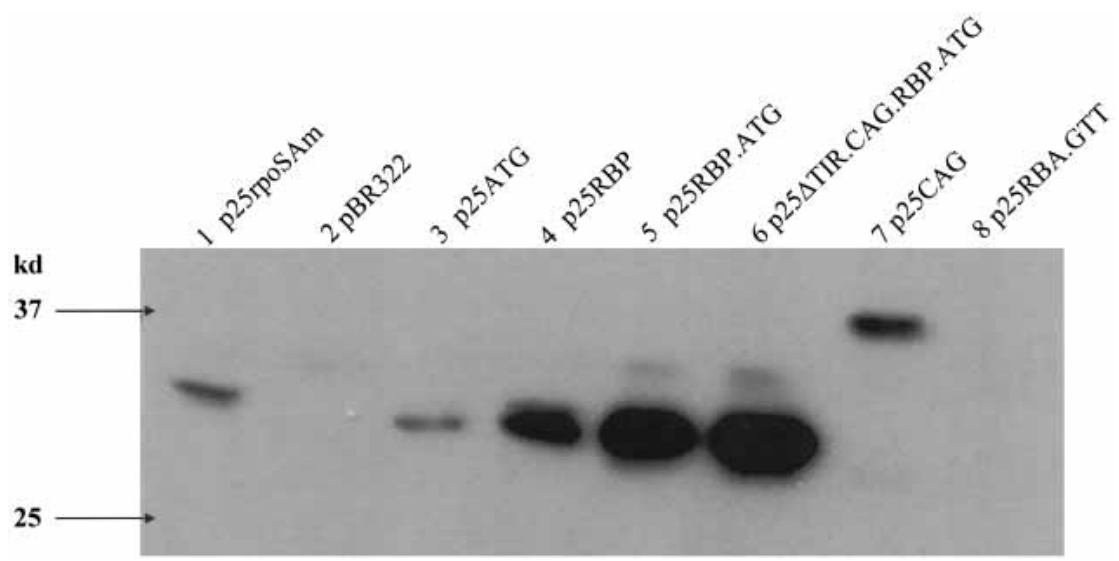

FIGURE 2. Mutations in the rpoS STIR affects its translation. $\Delta r p o S$ strain ZK1000 transformed with respective plasmids were grown in LBKan/Amp. Plasmid-borne rpoS was induced with $1.0 \mathrm{mM}$ IPTG. A total of 10-30 $\mu \mathrm{g}$ of the total cell lysate was resolved on a $12 \%$ polyacrylamide gel, electroblotted, and probed with anti- $\sigma^{\mathrm{S}}$ antibody. $\sigma^{\mathrm{S}}$-positive bands were scanned, and densitometric analyses were carried out using ImageQuant software (Molecular Dynamics). Western immunoassays were repeated multiple times.
Translation Initiation Region. This eliminates efficient recruitment of ribosomes by the STIR (Fig. 5A, below). However, in the event of premature translation termination as demonstrated in this work, STIR could reinitiate translation. Therefore, the failure to translate $\Delta 1-53 \sigma^{\mathrm{S}}$ from the plasmid $p 25 C A G$ highlights the dependence of the STIR on the presence of an upstream stop codon in particular or ribosome disassembly in general.

\section{Translation (re)initiation from STIR is independent of the primary TIR}

Each cistron of a polycistronic message may have an independent TIR. However, internal cistrons of some polycistronic messages do not have an independent TIR. The stop codon of upstream cistron and the initiator codon of the downstream cistron overlap. A functional ribosome, upon encountering the stop codon instead of disassembling, initiates translation of the downstream cistron. Thus, these two cistrons are translationally coupled. Defect in translation of the first cistron abolishes the translation from the second or the downstream cistron (Inokuchi et. al. 2000). E. coli rpoS is transcribed as a monocistronic message (Mukhopadhyay et. al. 2000) that contains two TIRs (Subbarayan and Sarkar 2004b). We wanted to check whether these two TIRs are coupled. By removing the primary TIR in p25CAG and perfecting the STIR, we created the mutant plasmid p25DTIR.CAG.RBP.ATG (Table 1). A $\Delta 1-53 \sigma^{\mathrm{S}}$-positive band corresponding to $\sim 30 \mathrm{kDa}$ was detected from the transformants of this construct (Fig. 2, lane 6). The catalase II activity was comparable to p25CAG/ZK1000 (Table 2). Activation of STIR in the absence of primary TIR as in p25 TIR.CAG.RBP.ATG demonstrates the ability of STIR to support translation initiation in the rpoSAm gene. Thus, our results conclusively demonstrate that STIR is not translationally coupled to TIR.

The degree of overexpression of $\Delta 1$ $53 \sigma^{\mathrm{S}}$ by different mutant constructs is compared in Table 2. Among all the mutants analyzed, the increase in the quantity of truncated $\sigma^{\mathrm{S}}$ and corresponding increase in their (catalase II) activity is not proportional (Table 2).

There is a competition among different $\sigma \mathrm{s}$ for the core RNA polymerase (Jishage et. al. 2002). Therefore, the molar ratios of available $\sigma$ s might determine the profile of the holoenzyme. Dr. Gowrishankar and his colleagues demonstrated that $\mathrm{N}$-terminal truncated $\sigma^{\mathrm{S}}$ is only partially functional on the katE promoter in vivo (Rajkumari and Gow- 
TABLE 2. Comparison of changes in $\Delta 1-54 \sigma^{\mathrm{s}}$ quantity and their corresponding activity as monitored by catalase assay in different mutants

\begin{tabular}{|c|c|c|c|c|}
\hline \multirow[b]{2}{*}{ S. no } & & \multirow{2}{*}{$\begin{array}{c}\text { Catalase II activity } \\
(\mu \mathrm{mole} / \mathrm{mg} / \mathrm{min})\end{array}$} & \multicolumn{2}{|c|}{ Fold changes in } \\
\hline & & & Catalase II activity & $\Delta 1-53 \sigma^{\mathrm{s}}$ quantity \\
\hline 1 & p25rpoSAm/p25rpoSAm & 1.34 & 1.00 & 1.00 \\
\hline 2 & p25ATG/p25rpoSAm & 2.14 & 1.60 & 2.46 \\
\hline 3 & p25RBP/p25rpoSAm & 6.34 & 4.70 & 11.29 \\
\hline 4 & p25RBP.ATG/p25rpoSAm & 13.65 & 10.2 & 20.20 \\
\hline 5 & p25 2 TIR.CAG.RBP.ATG/p25rpoSAm & 26.72 & 20.0 & 24.46 \\
\hline 6 & p25CAG/p25rpoSAm & 11.91 & 8.9 & 5.41 \\
\hline 7 & p25RBA.GTT/p25rpoSAm & 0.2 & 0.15 & - \\
\hline
\end{tabular}

Catalase assay results and densitometry readings of the Figure 2 are compared. Catalase assay results are average of at least three independent replicates.

rishankar 2002). Compared with that of p25rpoSAm, fulllength $\sigma^{\mathrm{S}}$ from $p 25 C A G$ and $\Delta 1-53 \sigma^{\mathrm{S}}$ from p25DTIR.CAG.RBP.ATG is synthesized five and 24 times more. Due to the difference in their quantity, presumably a large proportion of holoenzyme with the truncated $\sigma^{\mathrm{S}}$ is formed. The inefficient recognition of katE promoter by the holoenzyme containing the N-terminal-truncated $\sigma^{\mathrm{S}}$ may be offset by its number. Therefore, even though there is a big difference in $\sigma^{\mathrm{S}}$ quantity, the catalase activity in these two transformants seems identical.

\section{p25GTT and p25RBA.GTT are defective in translation and not transcription}

There is a possibility that by mutating the STIR region, we inadvertently affected transcription. To rule it out, the mutant constructs were transcribed in vitro under the control of a T7 promoter. All of the constructs, including p25GTT and $p 25 R B A . G T T$, in which the STIR region was abolished, supported full-length transcription (data not shown). In the coupled in vitro transcription/translation experiment, compared with p25rpoSAm, there is a $250 \%$ increase in translation from $p 25 A T G$, whereas the incorporation of $\left[{ }^{35} \mathrm{~S}\right] \mathrm{me}-$ thionine is nearly $(\sim 10 \%)$ abolished in p25GTT and p25RBA.GTT (Fig. 3). The $10 \%$ TCA precipitable radioactive incorporation may be due to premature translation termination at the amber codon 33. These experimental results established the transcriptional integrity of the mutant constructs. It also suggests that the amount of protein translated in these mutants is due to the role played by the STIR.

\section{W3350 (rposAm) exhibits GASP phenotype}

$\sigma^{\mathrm{S}}$ is a global transcriptional regulator. It is known that partial loss-of-function
$\sigma^{\mathrm{S}}$ mutants (rpoS819) confer growth advantage in stationary phase (GASP) phenotype (Zambrano and Kolter 1996). RpoSAm mutants also exhibit partial loss-of-function, as evidenced by reduced catalase activity. To examine whether rpoSAm possess GASP phenotype, we grew the rpoSAm strain W3350 in competition with ZK126, which expresses full-length $\sigma^{\mathrm{S}}$. Within days, the amber mutant W3350 dominated the culture, thus establishing its GASP phenotype over its wild-type counterpart (Fig. 4). Under the same experimental conditions, we could not detect any change in the bubbling phenotype in the parallel individual independent cultures. Their CFU stabilized in the range of $1 \times 10^{7} /$ $\mathrm{mL}$. We successfully reproduced the results of this competition assay under isogenic background using plasmidborne mutant constructs (data not shown).

Dr. Kolter's GASP mutants (rpoS819) carry a C-terminal extension, leading to its loss-of-function (Zambrano et. al. 1993). Amber stop is common among the different types of mutations reported in rpoS. Stop codon TAG was recently proposed to be the precursor of CAG at the same position

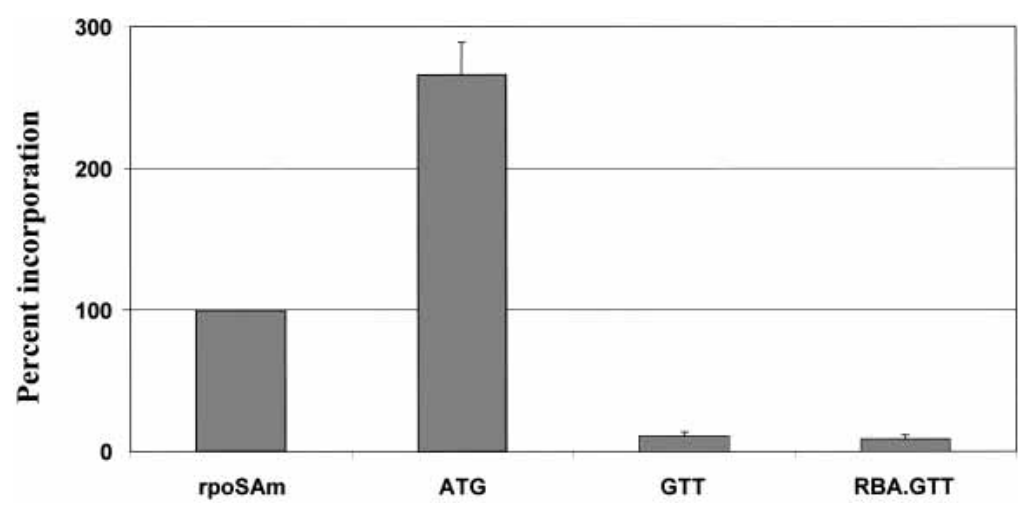

FIGURE 3. Mutations in STIR affect translation. A total of $2 \mu \mathrm{g}$ of linearized plasmids, viz., p25rpoSAm, p25ATG, p25GTT, and p25RBA.GTT were assayed for their translational efficiency. Each of the $25-\mu \mathrm{L}$ reaction mixtures contained the template, $\left[{ }^{35} \mathrm{~S}\right]$ Met and EcoPro Extract (Novagen) and was incubated at $37^{\circ} \mathrm{C}$ for $60 \mathrm{~min}$. At the end, TCA insoluble radioactivity was counted. The total TCA precipitable counts from the reaction mix with p25rpoSAm as the template was taken as $100 \%$ reference value. All of the experiments were repeated at least two times. 


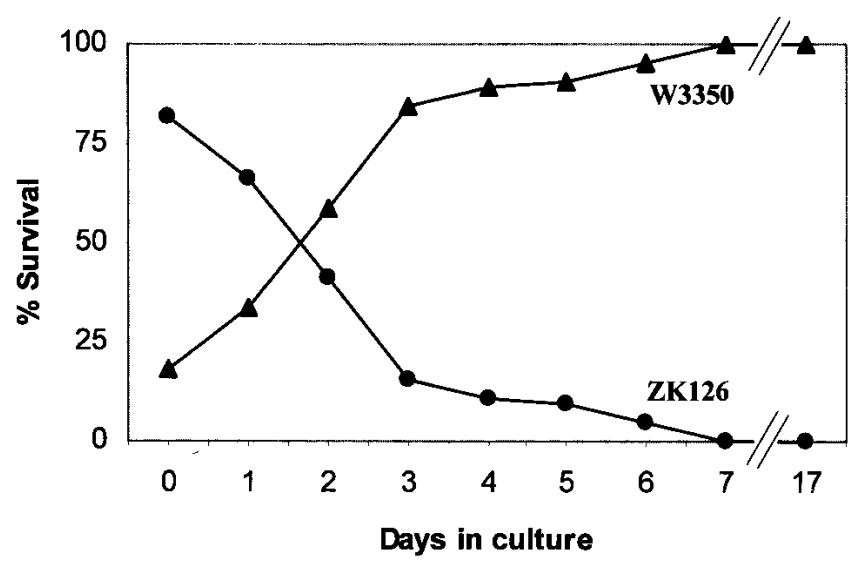

FIGURE 4. rpoSAm mutants have survival advantage. ZK126, wild type for rpoS and the rpoSAm strain W3350 were cocultured continually at $37^{\circ} \mathrm{C}$. Every $24 \mathrm{~h}$, the CFU of the culture was determined by overnight plating, followed by a simple catalase test to distinguish between the two strains. The total CFU of the cultures stabilized in the range of $10^{7}$. Competition assays were reproducibly repeated multiple times under different conditions, as well as isogenic background.

(Atlung et. al. 2002; Subbarayan and Sarkar 2004a). In this study, we established that the activation of STIR is contingent upon the presence of this particular stop codon. Results of our competition assay demonstrated GASP phenotype, and thereby the physiological sig-

nificance of STIR in rPoSAm strains.

\section{Which is the mutant: 330-amino-acid-long $\sigma^{\mathrm{s}}$ or $\Delta 1-53 \sigma^{\mathrm{s}}$ ?}

Both the 330-amino-acid-long $\sigma^{\mathrm{S}}$ and 277-amino-acid-long $\Delta 1-53 \sigma^{\mathrm{S}}$ are functional. In fact, a functional STIR confers survival advantage. rpoSAm is widespread among different strains and is also common of all the different types of mutations found in rpos. Recently, Atlung et. al. (2002) proposed amber codon TAG as the parental codon 33 in K-12 strains of E. coli rpoS and variants such as CAG, CTG, TAT, or TCG to be its derivatives. These facts raise serious concerns about the size of the full-length $\sigma^{\mathrm{S}}$ protein.

We speculate that in typical nutrientrich laboratory conditions, strains like MG1655, W3110, etc., with CAG as codon 33, might have successfully survived. On the other hand, rpoSAm strains would have benefited in a nutrient-limiting environment. MG1655 and W3110 have traditionally been used as reference or wild-type strains. Therefore, the rpoS gene, with CAG as codon 33 isolated from them, is naturally presumed to be the wild type. Literature is replete with rpoSAm mutants. It is difficult to account for the same mutation occurring at the same position in large number of strains. However, a few strains that have been determined to have CAG as codon 33 might be the mutants. In vivo and in vitro functional studies with $\Delta 1-50 \sigma^{\mathrm{S}}$ and $\Delta 1-53 \sigma^{\mathrm{S}}$, respectively (Rajkumari and Gowrishankar 2002; Gowrishankar et. al. 2003), the proposal of TAG as the parental codon 33 (Atlung et. al. 2002), demonstration of the functional STIR, and rpoSAm as GASP mutants make a strong case to redefine wild-type or the original $\sigma$ protein to be 277 amino acids long and the hitherto-known 330-residue-long $\sigma^{\mathrm{s}}$ to be the mutant protein.

\section{Working model for the STIR}

On the basis of our results, we detailed in Figure 5 conditions wherein the STIR could be activated. Normally, (Fig. $5 \mathrm{~A}$ ) unstalled ribosomes proceeding from a primary TIR overrides the STIR. On the other hand, the STIR is activated by two pathways, namely, ribosome disassembly ahead of the STIR as in rpoSAm mutants (Fig. 5B), or absence of

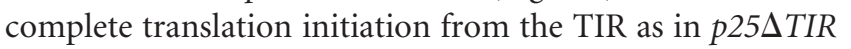
(Fig. 5C). In the absence of STIR, premature translation
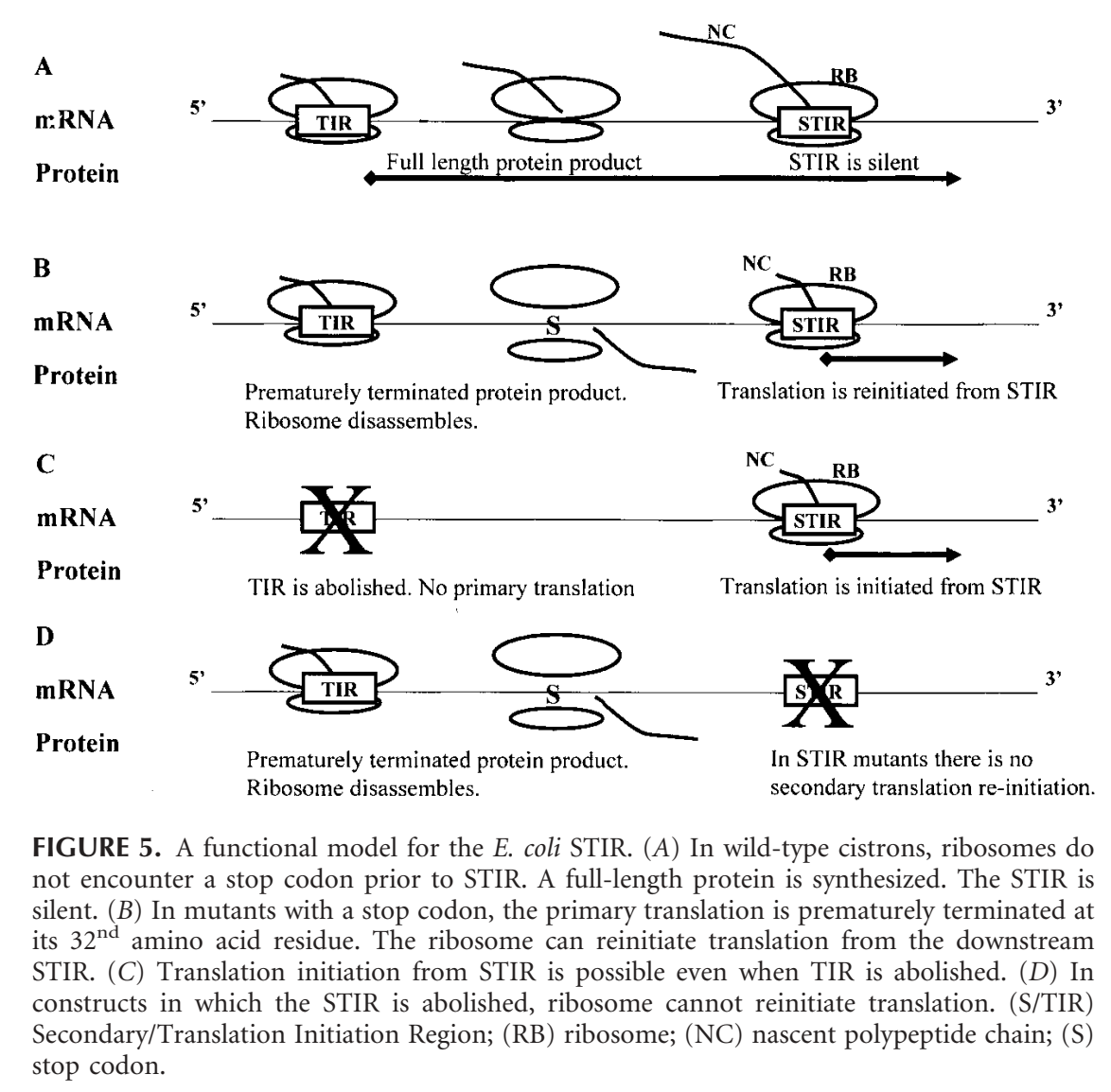
termination leads to loss of protein (Figs. 2,5D), consequently, catalase II activity (Table 2). The functional status of the protein synthesized by this kind of gene arrangement depends on the maintenance of the reading frame and the position of truncation and translation reinitiation. RpoSAm is a typical example in which the reading frame is maintained and the $\Delta 1-53 \sigma^{\mathrm{s}}$ retained its function (Gowrishankar et. al. 2003).

The principal outcome of our study is the confirmation of the existence of an independent internal STIR within an E. coli rpoS. The presence of internal ribosome entry sites (IRES) is known in eukaryotes, wherein the expression of a protein is regulated by translation initiation from different start sites from within the coding region (Cornelis et. al. 2000). E. coli CheA, infB, and IF2 $\beta$ genes have been reported to have intracistronic translation initiation or the STIR. However, proteins synthesized by intracistronic translation mode did not confer any physiological significance to their hosts (Smith and Parkinson 1980; Nyengaard et. al. 1991; Laursen et. al. 2002). Therefore, to our knowledge, our results for the first time describe a physiologically significant IRES equivalent in the E. coli rpoS gene.

\section{Conclusion}

Our work demonstrates the existence of translation reinitiation from the rpoS STIR, and the GASP nature of rpoSAm mutants.

\section{MATERIALS AND METHODS}

\section{Bacterial strains and culture conditions}

E. coli $\mathrm{K}-12(\lambda)$ strain ZK126 (W3110 tna-2 $\Delta$ lacU116) was considered wild type for these experiments. Strains ZK126 and ZK1000 (=ZK126 rpoS::kan) were a kind gift of Dr. R. Kolter (Harvard Medical School). RpoSAm strain W3350 (galK2, galT22, $\lambda^{-}$, IN(rrnD-rrnE) 1), was obtained from E. coli genetic stock center (Yale University). Cells were routinely grown at $37^{\circ} \mathrm{C}$ in Luria-Bertani medium (LB) or plates supplemented with appropriate antibiotics.

\section{Chemicals and enzymes}

Polyclonal anti- $\sigma^{\mathrm{s}}$ antibody was a kind gift of Dr. Kan Tanaka (Tokyo University). Oligonucleotides for PCR and DNA sequencing were prepared in the in-house facility. Site-directed mutagenesis kit and high-fidelity cloned Pfu DNA polymerase were purchased from Stratagene. All other chemicals were reagent grade.

\section{Plasmids for rpoS cloning and expression}

p25rpoSAm $\left(\mathrm{Amp}^{\mathrm{r}}\right.$; Subbarayan and Sarkar 2004b) is a pBR322based plasmid. Primers were designed with desired base changes. They were introduced to plasmids by PCR. The parental plasmids used as templates for PCR were fragmented with methylation- sensitive restriction enzyme DpnI. Thus, the PCR mix was left with newly synthesized, but intact unmethylated products. It was transformed to XL1-Blue Supercompetent cells (Stratagene) and plated onto LBAmp plates. A few colonies were screened and sequenced to confirm the introduced mutations. They were subsequently transformed to ZK1000 cells for further characterization. Successive multiple mutants were generated using single mutants as the template. For example, the initial/original plasmid p25rpoSAm was used to obtain the first set of mutants, such as $p 25 C A G, p 25 R B P$, p25ATG, p25GTT, p25RBA, etc. The double mutants such as p25ATG.RBP were obtained using $p 25 A T G$ or $p 25 R B P$ as the template. These processes were repeated to obtain the complete set of mutants. Quick Change Mutagenesis kit (Stratagene) was used for site-directed mutagenesis. The mutagenic primers used are listed in the Table 1. All of the PCR-mediated cloning steps, plasmid isolation, and transformation of plasmids were as per standard protocol (Sambrook and Russell 2001).

\section{Immunodetection of $\sigma^{\mathrm{s}}$}

To detect $\sigma^{\mathrm{S}}$ protein, the $\Delta r p o S$ strain ZK1000, transformed with p25rpoSAm or its mutant derivatives were grown to an $\mathrm{OD}_{600}$ of $\sim 0.3-0.5$. The rpoSAm expression was induced by the addition of IPTG to $1 \mathrm{mM}$, and the cells were harvested $3 \mathrm{~h}$ after induction. A total of 30 $\mu$ g cell lysates of ZK1000/p25rpoSAm, ZK1000/pBR322, and ZK1000/p25RBA.GTT, or $10 \mu \mathrm{g}$ of the rest of the transformants were electrophoresed on a $12 \%$ SDS-polyacrylamide gel, and then electroblotted onto a polyvinylidene fluoride membrane (PVDF; Millipore), using a semidry blotting system (Bio-Rad) and processed as per standard protocol. The immunoblot was scanned, and the anti- $\sigma^{\mathrm{S}}$-positive bands were quantitated using ImageQuant software (Molecular Dynamics). The degree of overexpression of the mutant $\sigma^{\mathrm{S}}$ is represented as the ratio of $\sigma^{\mathrm{S}}$ produced by the parental plasmid p25rpoSAm.

\section{Catalase assay}

Quantitation of catalase I (HPI) and catalase II (HPII) activities by spectrophotometry were carried out as described (Subbarayan and Sarkar 2004b).

\section{In vitro transcription/translation of p25rpoSAm and the mutants}

For in vitro transcription reaction, T7 promoter was introduced at the $5^{\prime}$ end of rpoS gene by PCR using the primer T7F $\left(5^{\prime}\right.$ GGATCCTAATACGACTCACTATAGGGCAGTGAGCGCAA-3'; underlined region is $\mathrm{T} 7$ promoter; the bold regions are complementary to the plasmid). This primer base paired $\sim 92 \mathrm{nt}$ upstream of the lac $\mathrm{P} / \mathrm{O}$ region in p25rpoSAm and its mutant derivatives. $R p o S$ gene from STIR mutants was synthesized by PCR using T7F as forward primer and T7R as reverse primer (5'-AGAATG GCAAAAGCTTACTCGCGGAACAGCGCTTCGAT-3'; bold italics is the stop codon, the bold region is the $3^{\prime}$ end of the rpoS) that complemented the $3^{\prime}$ end of the rpoS. The resultant PCR product of $1197 \mathrm{bp}$ was used for in vitro transcription using Large Scale T7 transcription kit (Novagen) as detailed in the product literature. A total of $10 \mu \mathrm{L}$ of the RNase free DNase I-treated in vitro-tran- 
scribed products were analyzed on $1 \%$ agarose gel and photographed.

Coupled in vitro transcription and translation was performed with $2 \mu \mathrm{g}$ of linearized p25rpoSAm, p25ATG, p25GTT, or p25RBA.GTT as template and $\left[{ }^{35} \mathrm{~S}\right]$ Met using EcoPro extract (Novagen) in a total reaction volume of $25 \mu \mathrm{L}$, as per the manufacturer's instructions. At the end, $10 \mu \mathrm{L}$ of reaction mixture was added to $0.9 \mathrm{~mL}$ of $25 \%$ TCA, and the TCA insoluble precipitant was counted in Beckman Liquid Scintillation counter, Model LS101. The total radioactive counts in p25rpoSAm precipitant were taken as $100 \%$ incorporation, and the rest of the results were compared against it.

\section{Coculture studies}

Overnight cultures of the E. coli strain ZK126 expressing the fulllength $\sigma^{\mathrm{S}}$ and $r p o S A m$ strain W3350 that expresses $\Delta 1-53 \sigma^{\mathrm{S}}(10 \mu \mathrm{L}$ each) were mixed in $3 \mathrm{~mL}$ of LB medium and incubated continuously at $37^{\circ} \mathrm{C}$. Every $24 \mathrm{~h}$, appropriate dilutions of the cultures were plated on LB plates to determine CFU. On an average, 95 colonies were tested for $\mathrm{H}_{2} \mathrm{O}_{2}$ bubbling phenotype. A total of 10 $\mu \mathrm{L}$ of $\mathrm{H}_{2} \mathrm{O}_{2}$ was dropped onto independent colonies, and its bubbling characteristic observed. ZK126 that carries an intact rpoS gene bubbled vigorously, whereas rpoSAm strain W3350 bubbled very slowly. Thus, the strains were discriminated on a single plate, and composition of the mixed culture was determined over a period of time. In parallel, CFU and the bubbling phenotype of ZK126 and W3350 cultured independently under identical conditions were also determined.

To check GASP phenotype in isogenic background, $10 \mu \mathrm{L}$ each of ZK1000/p25CAG and ZK1000/p25rpoSAm were cocultured in LB medium supplemented with Kan \& Amp. At 12-h post-inoculation, the medium was made $1 \mathrm{mM}$ with IPTG and continued culturing. Every $24 \mathrm{~h}$, an aliquot of the culture was withdrawn, and its composition determined as detailed in the earlier section.

\section{ACKNOWLEDGMENTS}

We thank Prof. M.P. Deutscher for his insightful guidance and encouragements. We acknowledge Dr. Rick Myers for his valuable comments and suggestions on translational coupling experiments. We thank Dr. N. Fujita for valuable discussion and his time. We also express our gratitude to Dr. Kan Tanaka for the anti $\sigma^{\mathrm{S}}$ antibody, and Drs. R. Kolter and Mary Berlyn for the E. coli strains used in this study.

The publication costs of this article were defrayed in part by payment of page charges. This article must therefore be hereby marked "advertisement" in accordance with 18 USC section 1734 solely to indicate this fact.

Received April 2, 2004; accepted June 22, 2004.

\section{REFERENCES}

Atlung, T., Nielsen, H.V., and Hansen, F.G. 2002. Characterization of the allelic variation in the rpoS gene in thirteen K12 and six other non-pathogenic Escherichia coli strains. Mol. Genet. Genomics 266: 873-881.

Barrick, D., Villanueba, K., Childs, J., Kalil, R., Schneider, T.D.,
Lawrence, C.E., Gold, L., and Stormo, G.D. 1994. Quantitative analysis of ribosome binding sites in E. coli. Nucleic Acids Res. 22: 1287-1295.

Cornelis, S., Bruynooghe, Y., Denecker, G., Van Huffel, S., Tinton, S., and Beyaert, R. 2000. Identification and characterization of a novel cell cycle-regulated internal ribosome entry site. Mol. Cell 5: 597605.

Gowrishankar, J., Yamamoto, K., Subbarayan, P.R., and Ishihama, A. 2003. In vitro properties of RpoS $(\sigma(S))$ mutants of Escherichia coli with postulated $\mathrm{N}$-terminal subregion 1.1 or $\mathrm{C}$-terminal region 4 deleted. J. Bacteriol. 185: 2673-2679.

Inokuchi, Y., Hirashima, A., Sekine, Y., Janosi, L., and Kaji, A. 2000. Role of ribosome recycling factor (RRF) in translational coupling. EMBO J. 19: 3788-3798.

Ishihama, A. 2000. Functional modulation of Escherichia coli RNA polymerase. Annu. Rev. Microbiol. 54: 499-518.

Jishage, M., Kvint, K., Shingler, V., and Nystrom, T. 2002. Regulation of $\sigma$ factor competition by the alarmone ppGpp. Genes \& Dev. 16: $1260-1270$.

Lange, R. and Hengge-Aronis, R. 1991. Identification of a central regulator of stationary-phase gene expression in Escherichia coli. Mol. Microbiol. 5: 49-59.

Laursen, B.S., de A. Steffensen, S.A., Hedegaard, J., Moreno, J.M., Mortensen, K.K., and Sperling-Petersen, H.U. 2002. Structural requirements of the mRNA for intracistronic translation initiation of the enterobacterial infB gene. Genes Cells 7: 901-910.

Mukhopadhyay, S., Audia, J.P., Roy, R.N., and Schellhorn, H.E. 2000. Transcriptional induction of the conserved alternative sigma factor RpoS in Escherichia coli is dependent on BarA, a probable twocomponent regulator. Mol. Microbiol. 37: 371-381.

Mulvey, M.R. and Loewen, P.C. 1989. Nucleotide sequence of katF of Escherichia coli suggests KatF protein is a novel $\sigma$ transcription factor. Nucleic Acids Res. 17: 9979-9991.

Nyengaard, N.R., Mortensen, K.K., Lassen, S.F., Hershey, J.W., and Sperling-Petersen, H.U. 1991. Tandem translation of E. coli initiation factor IF2 beta: Purification and characterization in vitro of two active forms. Biochem. Biophys. Res. Commun. 181: 15721579.

Parker, J. 1989. Errors and alternatives in reading the universal genetic code. Microbiol. Rev. 53: 273-298.

Rajkumari, K. and Gowrishankar, J. 2002. An N-terminally truncated RpoS (sigma(S)) protein in Escherichia coli is active in vivo and exhibits normal environmental regulation even in the absence of rpoS transcriptional and translational control signals. J. Bacteriol. 184: 3167-3175.

Sambrook, J. and Russell, D.W. 2001. Molecular cloning: A laboratory manual, 3rd ed. Cold Spring Harbor Laboratory Press, Cold Spring Harbor, NY.

Shine, J. and Dalgarno, L. 1975. Determinant of cistron specificity in bacterial ribosomes. Nature 254: 34-38.

Shultzaberger, R.K., Bucheimer, R.E., Rudd, K.E., and Schneider, T.D. 2001. Anatomy of Escherichia coli ribosome binding sites. J. Mol. Biol. 313: 215-228.

Smith, R.A. and Parkinson, J.S. 1980. Overlapping genes at the cheA locus of Escherichia coli. Proc. Natl. Acad. Sci. 77: 5370-5374.

Subbarayan, P.R. and Deutscher, M.P. 2001. Escherichia coli RNase M is a multiply altered form of RNase I. RNA 7: 1702-1707.

Subbarayan, P.R. and Sarkar, M. 2004a. A comparative study of variation in codon 33 of the rpoS gene in Escherichia coli K12 stocks: Implications for the synthesis of sigma(s). Mol. Genet. Genomics 270: 533-538.

- 2004b. Escherichia coli rpoS gene has an internal secondary translation initiation region. Biochem. Biophys. Res. Commun. 313: 294-299.

Zambrano, M.M and Kolter, R. 1996. GASPing for life in stationary phase. Cell 86: 181-184.

Zambrano, M.M., Siegele, D.A., Almiron, M., Tormo, A., and Kolter, R. 1993. Microbial competition: Escherichia coli mutants that take over stationary phase cultures. Science 259: 1757-1760. 

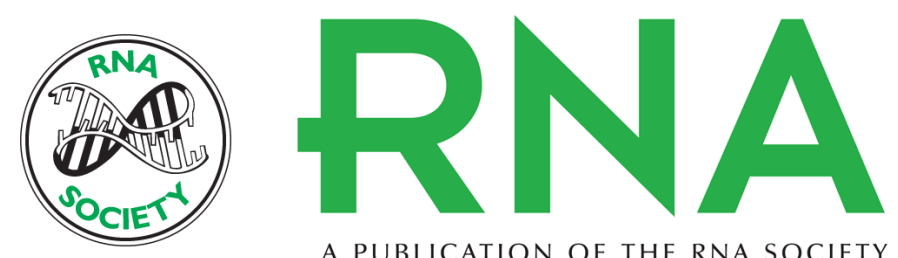

A PUBLICATION OF THE RNA SOCIETY

\section{A stop codon-dependent internal secondary translation initiation region in Escherichia coli rpos}

POCHI RAMALINGAM SUBBARAYAN and MALANCHA SARKAR

RNA 2004 10: 1359-1365

References This article cites 22 articles, 7 of which can be accessed free at:

http://rnajournal.cshlp.org/content/10/9/1359.full.html\#ref-list-1

\section{License}

Email Alerting

Receive free email alerts when new articles cite this article - sign up in the box at the Service top right corner of the article or click here. 